\section{MS39-P11 EIGER: The next generation of Hybrid Photon Counting detectors}

Marcus Mueller ${ }^{1}$, Arnau Casañas ${ }^{2}$, Meitian Wang ${ }^{2}$

1. DECTRIS Ltd., Switzerland

2. Swiss Light Source at Paul Scherrer Institut, Switzerland

email: marcus.mueller@dectris.com

PILATUS detectors have set new standards for data quality and collection efficiency in crystallographic data acquisition. EIGER, the next generation of Hybrid Photon Counting (HPC) detectors, takes a great leap towards smaller pixel size and substantially shorter readout times. The first EIGER detectors start operation at various synchrotron beamlines in 2015 and are also available for laboratory instruments.

This presentation will describe the novel features of EIGER and how crystallographers can benefit from these. Furthermore, first results obtained with EIGER detectors will be presented.

Keywords: Hybrid Pixel Detectors, X-ray detectors, Hybrid Photon Counting detectors

\section{MS39-P12 Application of a pnCCD for energy-dispersive Laue diffraction with ultra-hard X-rays}

Sebastian Send ${ }^{1}$, Ali Abboud ${ }^{1}$, Nadja Wiesner ${ }^{1}$, Mohammad Shokr $^{1}$, Tuba Conka-Nurdan ${ }^{2}$, Manuela Klaus ${ }^{3}$, Christoph Genzel $^{3}$, Dieter Schlosser ${ }^{4}$, Martin Huth ${ }^{4}$, Robert Hartmann ${ }^{4}$, Lothar Strüder ${ }^{4}$, Ullrich Pietsch ${ }^{1}$

1. University of Siegen, Germany

2. Türk-Alman Üniversitesi, Istanbul, Turkey

3. Helmholtz-Zentrum Berlin, Germany

4. PNSensor GmbH, Munich, Germany

email: send@physik.uni-siegen.de

The use of highly brilliant synchrotron radiation allows precise analyses of crystalline materials by means of diffraction experiments. However, in a variety of applications, the availability of structural information is limited due to the insufficient performance of conventional detector systems for X-ray spectroscopy. New experimental possibilities are offered by energy-resolving area detectors based on the concept of the pn-junction charge coupled device (pnCCD). In particular, structure determination can be achieved in a single-shot exposure of the crystal to white synchrotron radiation in an energy-dispersive Laue diffraction experiment. Until now pnCCDs have successfully been applied for analyses of inorganic crystals and fast screening techniques quantifying polycrystallinity in organic samples of macromolecules with white X-rays between $10 \mathrm{keV}$ and $35 \mathrm{keV}$.

In this work the spectroscopic performance of a pnCCD detector in the ultra-hard energy range between $40 \mathrm{keV}$ and $140 \mathrm{keV}$ is tested by means of an energy-dispersive Laue diffraction experiment on a GaAs crystal. About 100 Bragg peaks were collected in a single-shot exposure of the arbitrarily oriented sample to white synchrotron radiation provided by a wiggler and resolved in a large reciprocal-space volume. The positions and energies of individual Laue spots could be determined with a spatial accuracy of less than one pixel and a relative energy resolution below $1 \%$. In this way the unit-cell parameters of GaAs were extracted with high accuracy allowing for a complete indexing of the recorded Laue pattern. Experimental structure-factor amplitudes could be obtained from the three-dimensional data sets taking into account photoelectric absorption as well as Compton scattering processes inside the detector. The agreement between measured and theoretical kinematical structure-factor amplitudes calculated from the known crystal structure is in the range of $10 \%$. The results of this experiment demonstrate the potential of pnCCD detectors for applications in X-ray structure analysis using the complete energy spectrum of synchrotron radiation.

References:

S. Send et al., Nucl. Instrum. Meth. A, 711 (2013), 132-142

A. Abboud et al., J. Instrum. 8 (2013), P05005

S. Send et al., J. Appl. Cryst. 45 (2012), 517-522

S. Send et al., J. Appl. Cryst. 42 (2009), 1139-1146 


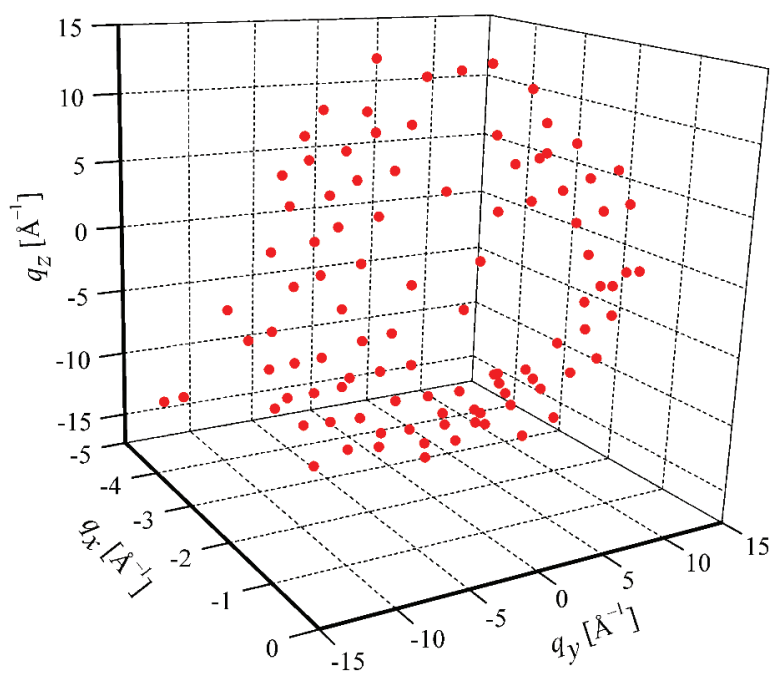

Figure 1. The recorded Laue pattern of GaAs after resolving the Laue spots' energies and converting the three-dimensional information into reciprocal-space coordinates. 101 Bragg peaks were collected in a single X-ray shot occupying reciprocal lattice points of GaAs in the defined coordinate system.

Keywords: energy-dispersive Laue diffraction, X-ray structure analysis, $\mathrm{X}$-ray spectroscopy, white synchrotron radiation, $\mathrm{pnCCD}$

\section{MS39-P13 The DIALS framework}

James M. Parkhurst ${ }^{1,2}$, Graeme Winter ${ }^{1}$, David Waterman ${ }^{3}$, Richard Gildea ${ }^{1}$, Luis Fuentes-Montero ${ }^{1}$, Gwyndaf Evans ${ }^{1}$

1. Diamond Light Source

2. MRC LMB

3. $\mathrm{CCP} 4$

email: james.parkhurst@diamond.ac.uk

Recent technological advances and changes in methodology in X-ray crystallography, such as high frame-rate pixel array detectors and serial femtosecond crytallography have created a need for the developement of new algorithms; however, current integration programs for X-ray diffraction data are limited by their lack of openness or extensibility, resulting in a barrier between the developement of new integration algorithms and their deployment on the beamline.

Here we present the DIALS (diffraction integration for advanced light sources) framework and integration package which is both a standalone application for the integration of X-ray diffraction data and a modular framework for developing new integration programs. The aim of the DIALS framework is to provide an extensible architecture within which new integration algorithms may be developed and quickly deployed without developers being required to write and maintain large amounts of high-level application source code. Rather, it is the aim of DIALS to liberate developers to allow them to concentrate soley on the scientific content of the algorithms themselves.

The DIALS framework implements a simple plugin system that allows algorithms to be loaded at runtime to perform certain tasks such as spot finding, indexing, refinement, background subtraction and integration. At the cost of writing a small amount of Python code, the developer can add an algorithm that can be loaded and selected at runtime without any modification of the DIALS framework itself. This design allows DIALS to be flexible with respect to the experiment being performed. For example, different algorithms can be used for data obtained using the rotation method or serial femtosecond crystallography.

DIALS provides a comprehensive suite of command line applications for the analysis of X-ray diffraction data as well as providing tools for visual inspection of the raw data and processing results. DIALS is written in a mixure of Python and $\mathrm{C}++$ and is available for download from http://sourceforge.net/projects/dials; the source code is available under an open-source BSD license.

Keywords: Integration, data processing, X-ray crystallography, software 